\title{
Ecandrewsite and zincian manganoan ilmenite from albitite veins of South Khetri Fold Belt, Rajasthan - implications in mineral search for basemetal-gold-uranium
}

Geological survey of India, Western Region, Jhalana Dungri, Jaipur-302004, India; *Corresponding author, E-mail: dbguha@gmail.com

(Received: August 7, 2016; Revised accepted: December 1, 2016)

http://dx.doi.org/10.18814/epiiugs/2017/v40i4/017032

Ecandrewsite mineral occurs within albitite vein intruded within the Udaipurwati granite gneiss from South Khetri Belt (SKB), Rajasthan, India. Microprobe analysis of the ecandrewsite gave compositional range from $\left(Z_{0.47} F_{0.34}\right.$

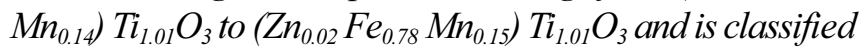
as ferroan manganoan ecandrewsite to zincian manganoan ilmenite. EPMA study reveals that the host rock consists of albite as major mineral constituent with some K-feldspar and chlorite as minor mineral constituents. The accessory minerals are sphene and ilmenite. On the basis of whole rock data, the albitite is classified as granite to syenite. The compositional variability within individual grains of ecandrewsite and other zinc bearing ilmenite suggests a replacement origin of the earlier formed magmatic mineral ilmenite. Core to rim enrichment of $Z n$ content within ecandrewsite suggest that migration of Zn along the grain boundaries of ilmenite occurred through mantle derived alkaline fluids from outside the system along the deep seated fracture zones and micro-shears; thus, precipitating many other metals (Cu, $A u, U, R E E)$ along with Zn. This association of ecandrewsite within albitite may be used as an indicator mineral for search of copper, gold, uranium and REE in cratonic parts of India and elsewhere in a similar geological set up.

\section{Introduction}

Ecandrewsite is a zinc bearing ilmenite and the mineral was first described from the Broken Hill lode in 1970 (New South Wales; Brown et al., 1970). Birch et al. (1988) studied the mineralogical details through EPMA, petrography and X-ray diffraction studies of the type samples of ecandrewsite and associated minerals from Broken Hill. Mitchell and Liferovich (2004) reported this unusual zincian manganoan ilmenite mineral from agpaitic nepheline syenites of South Africa and described paragenesis of this rare ilmenite structured titanates. Modification in chemistry of primary ilmenite is reported from many areas where the original magmatic ilmenite is replaced by $\mathrm{Mn}-\mathrm{Zn}$ bearing ilmenite during later processes (Sakoma and Martin, 2002).

The host rock containing the herein reported ecandrewsite and zincian manganoan ilmenite is predominantly albitite having mainly $\mathrm{Fe}$ Ti oxide studded albite (aventurine feldspar) with K-feldspar and chlorite as minor mineral constituents. Other accessory minerals are sphene and ilmenite. The occurrence of ecandrewsite and zincian manganoan ilmenite is not reported from the Khetri fold belt so far. The presence of this mineral is of importance because of its genetic implications with $\mathrm{Cu}-\mathrm{Au}-\mathrm{U}$ mineralisation in albitite as this mineral is found within undersaturated albitite associated with deep seated fracture zones within the ultramafic-mafic dominated basement in NW Indian craton. We report a new occurrence of ecandrewsite and zincian manganoan ilmenite from albitite veins intruded within Udaipurwati granite gneiss (Fig. 1) from South Khetri Fold Belt (SKB) having a variable empirical composition from $\left(\mathrm{Zn}_{0.47} \mathrm{Fe}_{0.34} \mathrm{Mn}_{0.14}\right) \mathrm{Ti}_{1.01} \mathrm{O}_{3}$ to $\left(\mathrm{Zn}_{0.02} \mathrm{Fe}_{0.78} \mathrm{Mn}_{0.15}\right) \mathrm{Ti}_{1.01} \mathrm{O}_{3}$, and its significance in mineral search in similar geological set up elsewhere.

\section{Geological Setting}

The Proterozoic Khetri fold Belt containing copper deposit in northern Rajasthan is cut across by a NW-SE trending Kantli fault into two halves; the North Khetri fold Belt and the South Khetri fold Belt. Both the belts show separate basement-cover relationship. The North Khetri fold belt is dominated by argillaceous-arenaceous facies rocks intruded by several granitic bodies, whereas the South Khetri fold Belt is dominated by felsic volcano-sedimentary rocks with plutonic granitic bodies such as Udaipurwati granite gneiss, Chhapoli granite, Seoli granite, etc. (Gupta et al., 1998). Occurrence of albitite in the Khetri fold Belt (Northern Rajasthan) defines a prominent crustal-scale linear petro-tectonic feature (Fig. 1), known as 'Albitite Line' which was first reported by Ray (1987, 1989 and 1990). The NNE-SSW trending albitite zone cuts across the Delhi and pre-Delhi rocks in the Khetri fold belt (KFB) (also known as Khetri copper belt (KCB) because of it containing known deposits of copper) and south of it, and broadly lies parallel to the trend of the Delhi orogeny. Before Ray (1987) it was called as aplite by Dasgupta, S.P. (1968). Ray (1990) further described this 'Albitite Line' as a fossil intracontinen- 


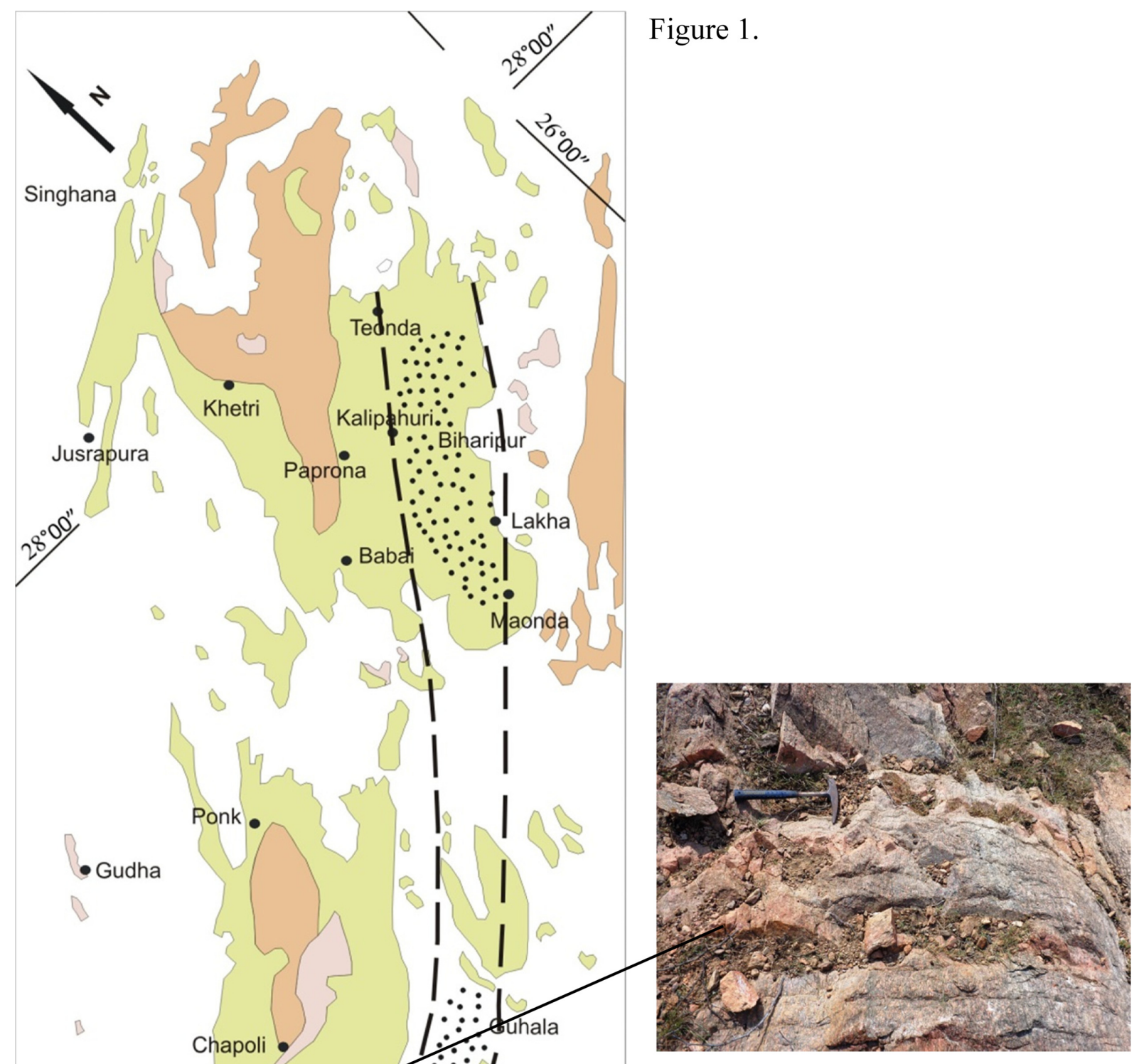

Figure 2.

Figures 1 (left) and 2 (right). Generalised geological map of the Khetri Fold Belt (KFB; after Dasgupta, 1968), showing location of albitite vein containing ecandrewsite. Dashed lines containing dots represent 'albitite line' of Ray (1990). 1 - albitite, 2 - granite gneiss, 3 - Ajabgarh Group, 4-Alwar Group. Field photograph of pink coloured albitite veins containing ecandrewsite, within Udaipurwati granite gneiss. 
tal rift zone. The majority of albitite occurrence was reported in the South Khetri fold belt where it occurs as vein, dykes and medium sized plugs associated with mafic, ultramafic and pelitic rocks along shear zones and deep seated fractures. During present work, we found small veins of albitite intruded within the Udaipurwati granite gneiss (Fig. 2) which lies $6 \mathrm{~km}$ west of 'Albitite line' in the SKB. Such albitite bodies $30 \mathrm{~km}$ west of the 'Albitite line' of Ray (op. cit.) are also reported from Arath area (Nagaur district) of NW Indian craton by Fareeduddin and Bose (1992) possibly indicating that the albitites do occur within the cratonic terrane away from the known 'Albitite line'. Occurrence of magnetite, ilmenite, chalcopyrite, pyrite and uraninite is reported from albitite by many other workers (Yadav et al., 2015) from $\mathrm{KCB}$ and the basement rocks (Banded gneissic complex, BGC) of Rajasthan. Guha and Mohanty (1994) reported albitite intruded within gneisses of the Mangalwar Complex (Bhilwara Supergroup, BSG) from Siana-Kajiawas area, farther away in this craton. Albitite is deep pink to brick red colour, composed of iron studded albite as major mineral, orthoclase and quartz as minor mineral and associated minerals are sphene, magnetite and ilmenite.

\section{Dataset and Sample Collection}

Total 21 samples were collected from different albitite bodies of SKB for major oxide, trace elements and REE analysis. 9 samples of albitite were collected from large dykes of the major albitite zone between Saladipura and Guhala (Fig. 1), and 12 samples were collected from the thin veins of albitite rock intruded within the Udaipurwati granite (Fig. 1). All the 21 samples were collected from fresh albitite rocks as grab samples which were not altered by weathering or later metasomatic alterations. For petrography 6 thin sections were studied from out of these 21 samples, and out of 6 samples studied by petrography, electron microprobe analysis were carried out for two samples which contained bigger grains of ilmenite as seen through petrographical study. For petrochemical studies, all samples thus collected have been analysed through XRF (for major oxides and trace elements) and ICPMS (for REE).

\section{Analytical Methods}

Electron microprobe analyses were carried out with a CAMECA, SX-100 microprobe at EPMA lab., Faridabad, GSI using an accelerating voltage of $15 \mathrm{kV}$ and a sample current of $10 \mathrm{nA}$. The beam size is $1 \mu$ and PIP correction factor was applied. X-ray fluorescence analyses for major oxide, trace elements and ICPMS analyses for rare earth elements (REE) were performed at Chemical laboratory GSI, WR, Jaipur, Rajasthan. The whole rock analysis through XRF was carried out to determine petrochemical variations in respect of samples collected from different bodies as explained earlier. The REE analysis through ICPMS was carried out to know anomalous values of REE for their petrogenetic correlations as the samples were collected from different bodies. EPMA of two samples were carried out to know the specific mineral composition of silicate and oxide minerals present in albitite rock of the area studied. XRD analysis was done to validate the EPMA data and also to know whether any other ilmenite bearing albitite sample also con- tained zincian ilmenite.

\section{Petrography and Mineral Chemistry}

In hand specimen albitite, containing ecandrewsite, is dark red to brick red in colour with dirty appearance. Under microscope it looks like a stained rock in the plane as well as cross polarised light and very difficult to identify the silicate minerals. However, albite the main constituent of this rock is iron studded albite with brown, cloudy appearance.

Ecandrewsite is brown to yellowish brown in colour (Fig. 3) in transmitted light and in reflected light, it is greyish white in colour (Fig. 4). Reflection pleochroism is weak but the mineral is strongly anisotropic with colours changing from greenish grey to dark brownish grey. Neither cleavage nor twinning is observed in thin polished section. Mineral phase analysis through Electron probe micro analyser (EPMA) study reveals that the albitite consists of albite as major mineral constituent with some $\mathrm{K}$-feldspar and chlorite as minor min-

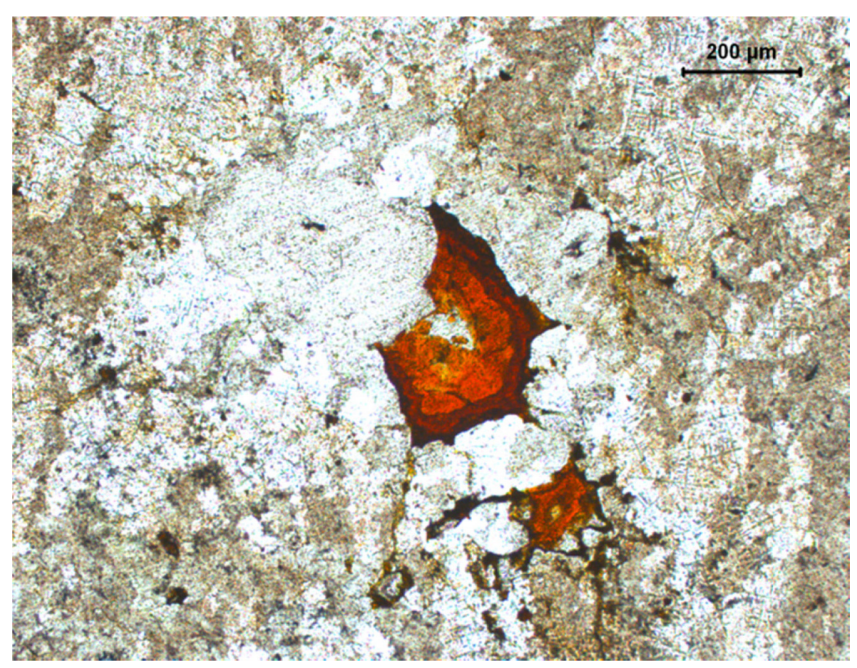

Figure 3. Photomicrograph of ecandrewsite in transmitted plane polarised light.

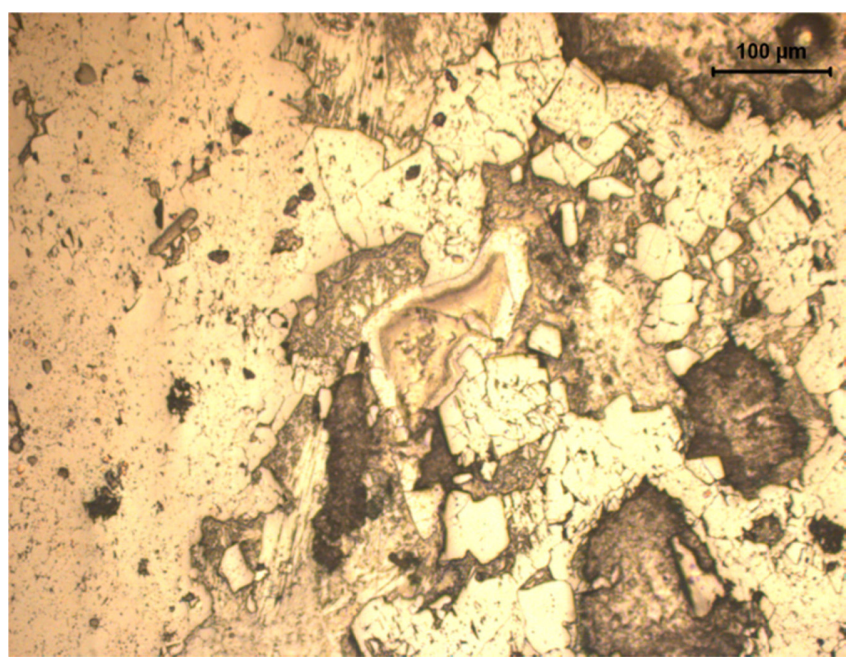

Figure 4. Photomicrograph of ecandrewsite in reflected plane polarised light. 
Table 1. Electron microprobe analysis data of Ecandrewsite and Zincian manganoan ilmenite (wt\%)

\begin{tabular}{|c|c|c|c|c|c|}
\hline Point no. & $\mathrm{TiO}_{2}$ & $\mathrm{FeO}$ & $\mathrm{ZnO}$ & $\mathrm{MnO}$ & Total \\
\hline 1 & 51.052 & 15.489 & 24.318 & 6.622 & 97.481 \\
\hline 2 & 49.584 & 17.94 & 19.38 & 6.249 & 93.153 \\
\hline 3 & 51.165 & 35.518 & 1.383 & 6.743 & 94.809 \\
\hline 4 & 60.036 & 18.714 & 6.638 & 1.095 & 86.483 \\
\hline 5 & 50.491 & 21.869 & 16.663 & 5.913 & 94.936 \\
\hline 6 & 47.306 & 21.293 & 16.051 & 6.015 & 90.665 \\
\hline 7 & 56.875 & 19.057 & 6.435 & 1.309 & 83.676 \\
\hline 8 & 49.852 & 15.714 & 22.485 & 6.248 & 94.299 \\
\hline 9 & 49.952 & 37.401 & 5.17 & 3.14 & 95.663 \\
\hline \multicolumn{7}{|c|}{$\mathrm{Cations} \mathrm{calculated} \mathrm{on} \mathrm{the} \mathrm{basis} \mathrm{of} \mathrm{3} \mathrm{oxygen} \mathrm{atoms}$} \\
\hline 1 & $\mathrm{Ti}$ & $\mathrm{Fe}$ & $\mathrm{Zn}$ & $\mathrm{Mn}$ & \\
\hline 2 & 1.01 & 0.34 & 0.47 & 0.14 & \\
\hline 3 & 1.02 & 0.41 & 0.49 & 0.14 & \\
\hline 4 & 1.2 & 0.41 & 0.13 & 0.02 & \\
\hline 5 & 1.02 & 0.49 & 0.33 & 0.13 & \\
\hline 6 & 1.007 & 0.5 & 0.33 & 0.14 & \\
\hline 7 & 1.19 & 0.44 & 0.13 & 0.03 & \\
\hline 8 & 1.02 & 0.35 & 0.45 & 0.14 & \\
\hline 9 & 0.99 & 0.83 & 0.1 & 0.07 & \\
\hline
\end{tabular}

Formula on the basis of 3 oxygen atoms are as follows:

$\left(\mathrm{Zn}_{0.47} \mathrm{Fe}_{0.34} \mathrm{Mn}_{0.14}\right) \mathrm{Ti}_{1.01} \mathrm{O}_{3}$

$\left(\mathrm{Zn}_{0.49} \mathrm{Fe}_{0.41} \mathrm{Mn}_{0.14}\right) \mathrm{Ti}_{1.02} \mathrm{O}_{3}$

$\left(\mathrm{Zn}_{0.02} \mathrm{Fe}_{0.78} \mathrm{Mn}_{0.15}\right) \mathrm{Ti}_{1.01} \mathrm{O}_{3}$

$\left(\mathrm{Zn}_{0.13} \mathrm{Fe}_{0.41} \mathrm{Mn}_{0.02}\right) \mathrm{Ti}_{1.2} \mathrm{O}_{3}$

$\left(\mathrm{Zn}_{0.33} \mathrm{Fe}_{0.49} \mathrm{Mn}_{0.13}\right) \mathrm{Ti}_{1.02} \mathrm{O}_{3}$

$\left(\mathrm{Zn}_{0.33} \mathrm{Fe}_{0.5} \mathrm{Mn}_{0.14}\right) \mathrm{Ti}_{1.0} \mathrm{O}_{3}$

$\left(\mathrm{Zn}_{0.13} \mathrm{Fe}_{0.44} \mathrm{Mn}_{0.03}\right) \mathrm{Ti}_{1.19} \mathrm{O}_{3}$

$\left(\mathrm{Zn}_{0.45} \mathrm{Fe}_{0.83} \mathrm{Mn}_{0.07}\right) \mathrm{Ti}_{0.99} \mathrm{O}_{3}$

$\left(\mathrm{Zn}_{0.10} \mathrm{Fe}_{0.44} \mathrm{Mn}_{0.03}\right) \mathrm{Ti}_{1.19} \mathrm{O}_{3}$

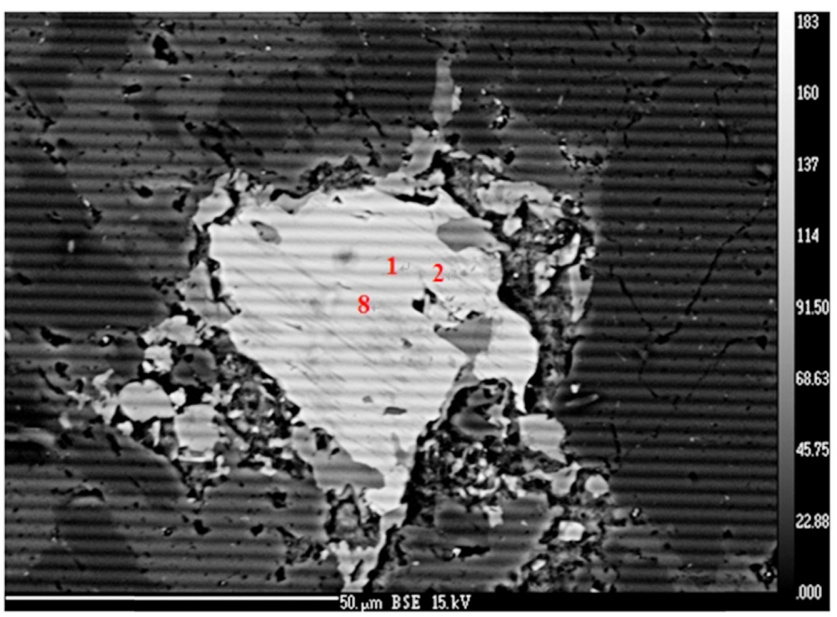

Figure 5. BSE image of ecandrewsite, the numbers in the image are the points which were analysed.

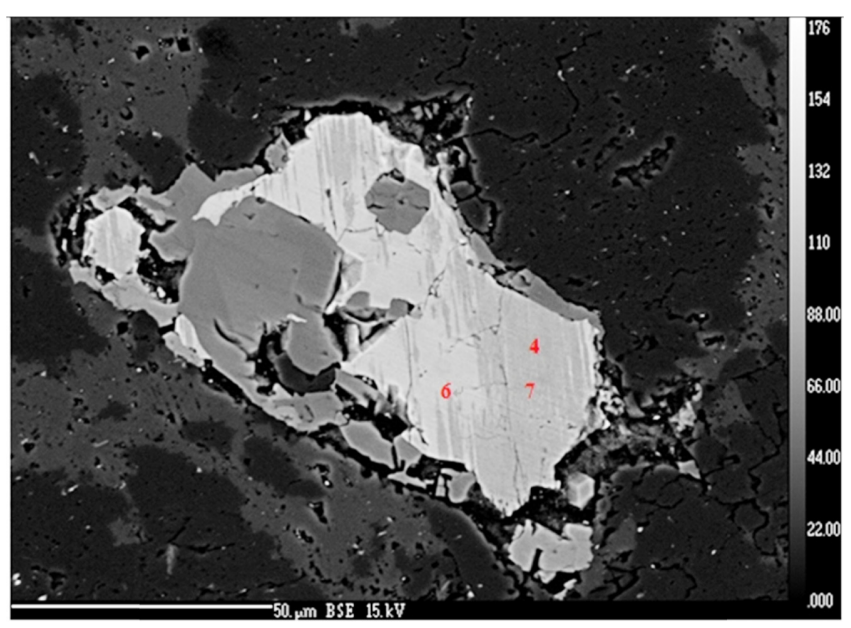

Figure 6. BSE image of zincian manganoan ilmenite, the numbers in the image are the points which were analysed.

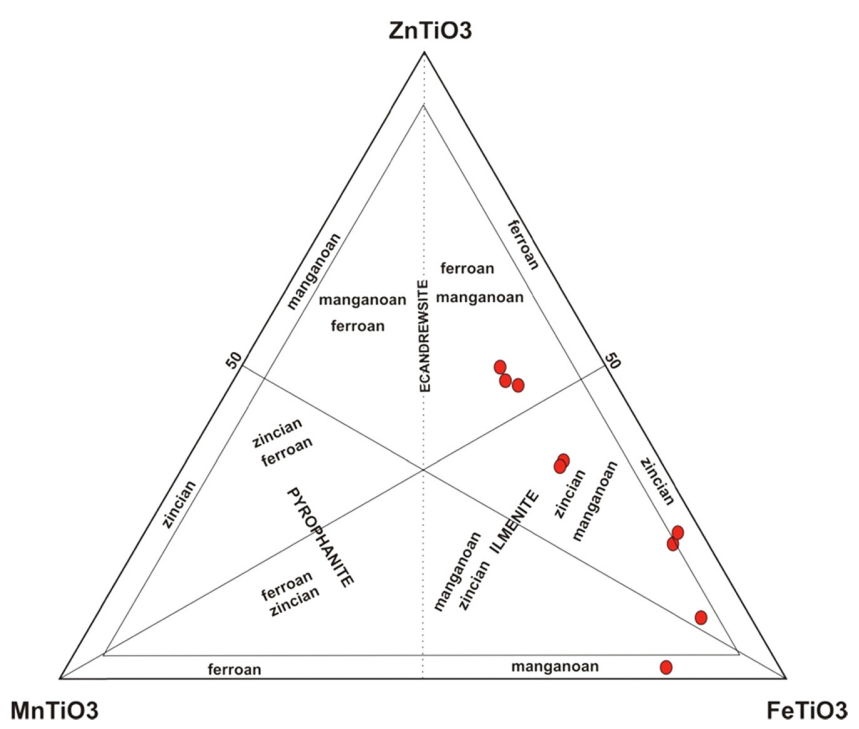

Figure 7. Classification of ecandrewsite and zinc bearing ilmenite in triangular diagram with three end members $\mathrm{ZnTiO}_{3}-\mathrm{FeTiO}_{3}$ $\mathrm{MnTiO}_{3}$ after Mitchell and Liferovich (2004).

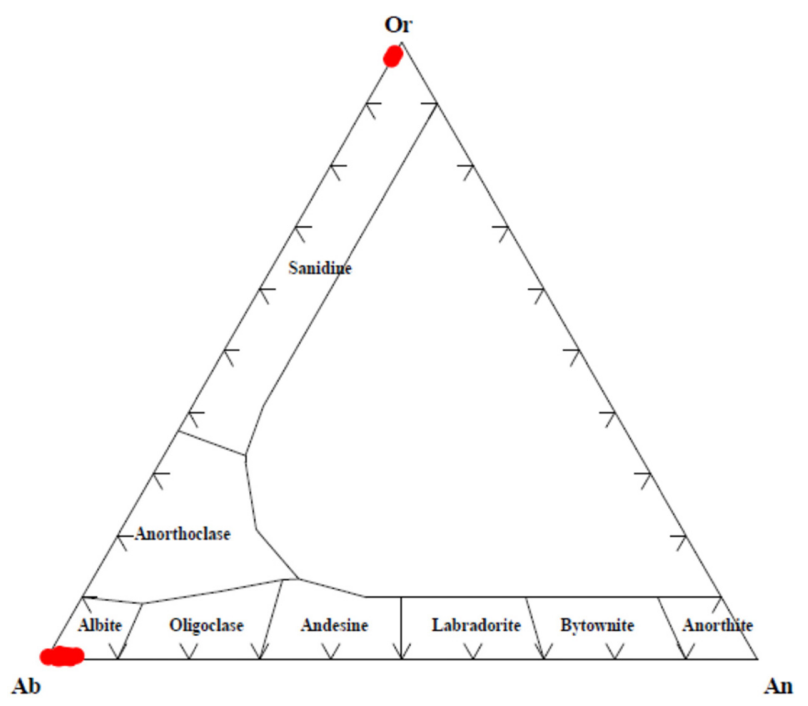

Figure 8. Classification of feldspars after calculating cations of albitite. 
Table 2. Major oxide (wt\%), trace elements (ppm), REE data (ppm) and normative minerals of albitite dykes from Saladipura-Guhala area

\begin{tabular}{|c|c|c|c|c|c|c|c|c|c|}
\hline & BRS-12 & BRS-13 & BRS-14 & BRS-18 & BRS-25 & BRS-26 & BRS-27 & BRS-28 & BRS-29 \\
\hline $\mathrm{SiO}_{2}$ & 70.88 & 62.07 & 76.96 & 71.44 & 72.89 & 74.96 & 63.58 & 76.26 & 75.84 \\
\hline $\mathrm{Al}_{2} \mathrm{O}_{3}$ & 10.94 & 14.49 & 10.75 & 12.63 & 11.61 & 11.6 & 14.83 & 11.44 & 9.77 \\
\hline $\mathrm{Fe}_{2} \mathrm{O}_{3}$ & 2.96 & 8.62 & 0.86 & 1.18 & 2.18 & 1.3 & 4.7 & 2.17 & 1.8 \\
\hline $\mathrm{MnO}$ & 0.02 & 0.04 & 0.01 & 0.06 & 0.01 & 0.01 & 0.07 & 0.01 & 0.01 \\
\hline $\mathrm{MgO}$ & 0.44 & 0.91 & 0.25 & 0.29 & 0.11 & 0.28 & 2.63 & 0.37 & 0.11 \\
\hline $\mathrm{CaO}$ & 2.43 & 1.71 & 1.08 & 1.39 & 2.37 & 0.34 & 2.25 & 0.35 & 0.92 \\
\hline $\mathrm{Na}_{2} \mathrm{O}$ & 6.56 & 1.7 & 5.81 & 7.66 & 8.43 & 8.79 & 8.91 & 7.38 & 6.43 \\
\hline $\mathrm{K}_{2} \mathrm{O}$ & 0.05 & 4.19 & 0.35 & 0.03 & 0.01 & 0.01 & 0.04 & 0.01 & 0.01 \\
\hline $\mathrm{TiO}_{2}$ & 0.49 & 0.88 & 0.07 & 0.56 & 0.33 & 0.17 & 1.14 & 0.39 & 0.33 \\
\hline $\mathrm{P}_{2} \mathrm{O}_{5}$ & 0.26 & 0.27 & 0.25 & 0.34 & 0.02 & 0.09 & 0.05 & 0.1 & 0.11 \\
\hline $\mathrm{Ba}$ & 2169 & 942 & $<50$ & 133 & 116 & $<50$ & 163 & 230 & 180 \\
\hline $\mathrm{Ga}$ & 13 & 14 & 2 & 20 & 17 & 21 & 21 & 11 & 6 \\
\hline $\mathrm{Sc}$ & 10 & 17 & 6 & 16 & 12 & 6 & 22 & 12 & 12 \\
\hline $\mathrm{V}$ & 33 & 83 & 14 & 25 & 24 & 17 & 70 & 27 & 23 \\
\hline $\mathrm{Th}$ & 139 & 76 & 12 & 34 & 32 & 27 & 27 & 42 & 36 \\
\hline $\mathrm{Pb}$ & 7 & 8 & $<5$ & 4 & $<5$ & 8 & 14 & $<5$ & $<5$ \\
\hline $\mathrm{Ni}$ & 14 & 11 & 7 & 12 & 8 & 7 & 132 & 6 & 6 \\
\hline $\mathrm{Co}$ & 8 & 16 & 8 & 5 & 14 & $<5$ & 76 & 9 & 7 \\
\hline $\mathrm{Rb}$ & 17 & 181 & 35 & 16 & 12 & 11 & 15 & 15 & 15 \\
\hline $\mathrm{Cr}$ & 42 & 10 & $<5$ & 5 & 13 & 11 & 172 & 22 & 6 \\
\hline $\mathrm{Sr}$ & 59 & 107 & 110 & 37 & 22 & 23 & 38 & 21 & 22 \\
\hline $\mathrm{Zr}$ & 485 & 740 & 40 & 415 & 493 & 446 & 180 & 169 & 220 \\
\hline $\mathrm{Nb}$ & 5 & 39 & $<5$ & 15 & 14 & $<5$ & 19 & 6 & 5 \\
\hline $\mathrm{Cu}$ & 2 & 28 & 20 & 13 & 18 & 35 & 459 & 34 & 26 \\
\hline $\mathrm{Zn}$ & 19 & 36 & 16 & 39 & 34 & 34 & 418 & 37 & 31 \\
\hline $\mathrm{Y}$ & 86.97 & 135.38 & 9.61 & 69.04 & 35.10 & 10.24 & 40.58 & 24.60 & 27.71 \\
\hline $\mathrm{U}$ & 5.45 & 4.72 & 2.98 & 3.46 & 6.40 & 7.99 & 8.98 & 15.73 & 13.32 \\
\hline $\mathrm{La}$ & 1977.74 & 233.04 & 48.13 & 167.00 & 123.80 & 53.73 & 69.67 & 114.20 & 143.30 \\
\hline $\mathrm{Ce}$ & 2593.74 & 421.36 & 56.48 & 193.30 & 209.00 & 94.86 & 138.20 & 313.20 & 242.10 \\
\hline $\operatorname{Pr}$ & 237.29 & 51.16 & 4.69 & 28.76 & 19.50 & 8.61 & 11.64 & 17.74 & 20.83 \\
\hline $\mathrm{Nd}$ & 878.01 & 190.32 & 15.95 & 107.40 & 66.90 & 28.43 & 41.82 & 56.57 & 67.49 \\
\hline $\mathrm{Sm}$ & 87.02 & 33.04 & 2.48 & 17.10 & 8.90 & 3.65 & 7.17 & 8.37 & 8.77 \\
\hline $\mathrm{Eu}$ & 9.07 & 4.56 & 0.94 & 2.44 & 1.80 & 0.67 & 1.25 & 1.23 & 1.16 \\
\hline $\mathrm{Gd}$ & 60.94 & 28.29 & 2.40 & 15.08 & 7.59 & 3.11 & 7.07 & 7.57 & 7.71 \\
\hline $\mathrm{Tb}$ & 5.45 & 4.91 & 0.39 & 2.42 & 1.11 & 0.38 & 1.20 & 1.04 & 1.05 \\
\hline Dy & 14.26 & 25.74 & 1.98 & 12.48 & 5.47 & 1.82 & 6.92 & 5.08 & 5.25 \\
\hline Ho & 2.30 & 5.52 & 0.39 & 2.69 & 1.26 & 0.38 & 1.50 & 1.03 & 1.11 \\
\hline $\mathrm{Er}$ & 7.10 & 15.32 & 1.07 & 7.33 & 3.57 & 1.14 & 4.23 & 2.79 & 3.05 \\
\hline $\mathrm{Tm}$ & 0.70 & 2.30 & 0.17 & 1.12 & 0.56 & 0.18 & 0.65 & 0.43 & 0.44 \\
\hline $\mathrm{Yb}$ & 4.42 & 14.64 & 1.19 & 6.96 & 3.61 & 1.19 & 3.97 & 2.70 & 2.82 \\
\hline $\mathrm{Lu}$ & 0.64 & 2.07 & 0.16 & 0.98 & 0.52 & 0.20 & 0.55 & 0.37 & 0.40 \\
\hline
\end{tabular}


Table 2. (continued)

\begin{tabular}{|c|c|c|c|c|c|c|c|c|c|}
\hline \multicolumn{10}{|c|}{ Normative minerals } \\
\hline & BRS-12 & BRS-13 & BRS-14 & BRS-18 & BRS-25 & BRS-26 & BRS-27 & BRS-28 & BRS-29 \\
\hline Q & 29.583 & 31.886 & 40.170 & 25.332 & 25.367 & 30.131 & 5.264 & 33.348 & 38.371 \\
\hline $\mathrm{C}$ & 0.000 & 4.695 & 0 & 0 & 0 & 0 & 0 & 0 & 0 \\
\hline Or & 0.295 & 24.762 & 2.068 & 0.177 & 0.059 & 0.059 & 0.236 & 0.059 & 0.059 \\
\hline $\mathrm{Ab}$ & 55.509 & 14.385 & 49.163 & 64.797 & 59.662 & 59.610 & 75.394 & 58.787 & 50.197 \\
\hline An & 0.257 & 6.720 & 2.219 & 0 & 0 & 0 & 0.352 & 0 & 0 \\
\hline $\mathrm{Ne}$ & 0 & 0 & 0 & 0 & 0 & 0 & 0 & 0 & 0 \\
\hline Ac & 0 & 0 & 0 & 0.018 & 6.307 & 3.761 & 0 & 3.225 & 3.710 \\
\hline Ns & 0 & 0 & 0 & 0 & 1.050 & 2.444 & 0 & 0 & 0 \\
\hline Di & 2.364 & 0 & 1.013 & 1.558 & 0.591 & 0.425 & 5.283 & 0 & 0.591 \\
\hline Wo & 2.269 & 0 & 0 & 0.400 & 4.074 & 0 & 0 & 0 & 0.825 \\
\hline Hy & 0 & 2.267 & 0.153 & 0 & 0 & 0.500 & 4.102 & 0.922 & 0 \\
\hline $\mathrm{Ol}$ & 0 & 0 & 0 & 0 & 0 & 0 & 0 & 0 & 0 \\
\hline Il & 0.043 & 0.086 & 0.021 & 0.128 & 0.021 & 0.021 & 0.150 & 0.021 & 0.021 \\
\hline $\mathrm{Hm}$ & 2.960 & 8.620 & 0.860 & 1.174 & 0 & 0 & 4.700 & 1.055 & 0.518 \\
\hline $\mathrm{Tn}$ & 1.148 & 0 & 0.144 & 1.209 & 0.782 & 0.390 & 2.605 & 0.763 & 0.782 \\
\hline Pf & 0 & 0 & 0 & 0 & 0 & 0 & 0 & 0 & 0 \\
\hline $\mathrm{Ru}$ & 0 & 0.835 & 0 & 0 & 0 & 0 & 0 & 0.068 & 0 \\
\hline Ap & 0.616 & 0.640 & 0.592 & 0.805 & 0.047 & 0.213 & 0.118 & 0.237 & 0.261 \\
\hline Sum & 95.044 & 94.895 & 96.403 & 95.599 & 97.961 & 97.555 & 98.204 & 98.485 & 95.336 \\
\hline
\end{tabular}

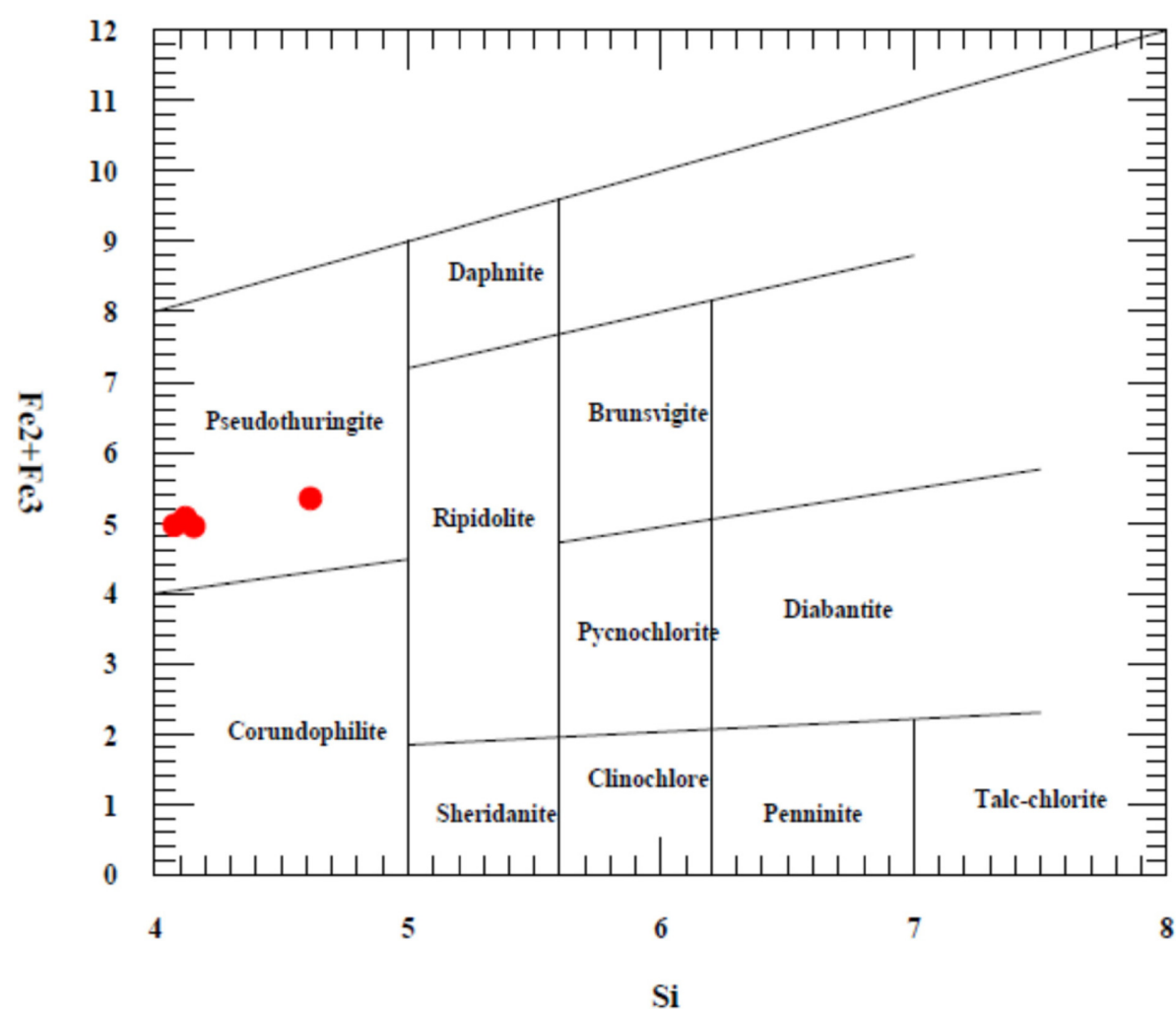

Figure 9. Classification of chlorite as pseudothuringite after calculating cations. eral constituents. The accessory minerals are sphene and ilmenite. Ecandrewsite present in albitite is associated with zincian manganoan ilmenite and they both are surrounded by sphene. EPMA results of ecandrewsite are given in the (Table 1). Zinc contents (Table 1, Figs. 5 and 6) are variable with maximum record being $24 \%$. The empirical formulae, based on 3 oxygen atoms, are also shown in Table 1. After calculating mineral formula, the minerals are classified in triangular diagram with three end members (Fig. 7). Considering the ideal formula for ecandrewsite as $\mathrm{ZnTiO}_{3}$, the composition ranges from $\left(\mathrm{Zn}_{0.47} \mathrm{Fe}_{0.34}\right.$ $\left.\mathrm{Mn}_{0.14}\right) \mathrm{Ti}_{1.01} \mathrm{O}_{3}$ to $\left(\mathrm{Zn}_{0.02} \mathrm{Fe}_{0.78} \mathrm{Mn}_{0.15}\right) \mathrm{Ti}_{1.01} \mathrm{O}_{3}$, and hence, they are ferroan manganoan ecandrewsite to zincian manganoan ilmenite. The EPMA analyses of the other silicate minerals are given in the Table 2 . The analyzed feldspar and chlorites were plotted in mineral classification diagrams. Majority of the feldspar 8 are albite (Fig. 8) having compositional ranges $\mathrm{Ab}_{99.6} \mathrm{An}_{0.1} \mathrm{Or}_{0.3}$ to $\mathrm{Ab}_{95.6} \mathrm{An}_{3.9} \mathrm{Or}_{0.5}$ with very minor orthoclase. Chlorites are classified as pseudothuringite (Fig. 9). 
Table 3. Major oxide (wt\%), trace elements (ppm), REE data (ppm) normative minerals of thin veins of albitite intruded within Udaipurwati granite

\begin{tabular}{|c|c|c|c|c|c|c|c|c|c|c|c|c|}
\hline & BRS-4 & BRS-46 & BRS-47 & BRS-86 & BRS-90 & BRS-91 & BRS-92 & BRS-100 & BRS-101 & BRS-102 & BRS-103 & BRS-84 \\
\hline $\mathrm{SiO}_{2}$ & 77.64 & 71.25 & 68.4 & 72.1 & 67.53 & 60.61 & 74.36 & 72.95 & 60.23 & 64.55 & 56.13 & 72.12 \\
\hline $\mathrm{Al}_{2} \mathrm{O}_{3}$ & 11.43 & 11.68 & 13.92 & 14.21 & 16.11 & 18.34 & 13.34 & 13.37 & 14.47 & 13.88 & 21.59 & 14.41 \\
\hline $\mathrm{Fe}_{2} \mathrm{O}_{3}$ & 0.86 & 3.99 & 4.48 & 1.24 & 2.12 & 4.56 & 0.47 & 1.88 & 2.62 & 2.08 & 2.62 & 1.3 \\
\hline $\mathrm{MnO}$ & 0.03 & 0.03 & 0.05 & 0.02 & 0.03 & 0.04 & 0 & 0.02 & 0.03 & 0.03 & 0.1 & 0.02 \\
\hline $\mathrm{MgO}$ & 0.11 & 0.4 & 0.44 & 0.19 & 0.16 & 0.37 & 0.03 & 0.33 & 3.35 & 2.38 & 0.31 & 0.2 \\
\hline $\mathrm{CaO}$ & 0.48 & 0.84 & 1.09 & 0.43 & 0.56 & 0.38 & 0.18 & 0.36 & 4.92 & 4.56 & 0.74 & 0.44 \\
\hline $\mathrm{Na}_{2} \mathrm{O}$ & 5.33 & 2.83 & 3.60 & 7.26 & 7.26 & 9.46 & 8.44 & 4.39 & 9.3 & 8.88 & 11.28 & 7.44 \\
\hline $\mathrm{K}_{2} \mathrm{O}$ & 1.03 & 5.85 & 5.33 & 2.29 & 3.87 & 2.62 & 1.39 & 3.92 & 0.25 & 0.27 & 1.13 & 2.34 \\
\hline $\mathrm{TiO}_{2}$ & 0.27 & 0.38 & 0.46 & 0.08 & 0.33 & 0.48 & 0.25 & 0.43 & 0.67 & 0.66 & 0.49 & 0.08 \\
\hline $\mathrm{P}_{2} \mathrm{O}_{5}$ & 0.08 & 0.08 & 0.13 & 0.04 & 0.11 & 0.03 & 0.03 & 0.09 & 0.12 & 0.11 & 0.17 & 0.04 \\
\hline $\mathrm{Ba}$ & 50 & 350 & 367 & 24 & 383 & 157 & 42 & 410 & 17 & 67 & 238 & 26 \\
\hline $\mathrm{Ga}$ & 23 & 30 & 34 & 24 & 6 & 5 & 7 & 5 & 19 & 12 & 31 & 26 \\
\hline $\mathrm{Sc}$ & 5 & 6 & 7 & 3 & 3 & 7 & 5 & 6 & 20 & 14 & 10 & 4 \\
\hline $\mathrm{V}$ & 14 & 34 & 40 & 15 & 24 & 39 & 17 & 25 & 34 & 32 & 29 & 15 \\
\hline Th & 92 & 92 & 116 & 77 & 96 & 81 & 102 & 85 & 21 & 18 & 92 & 77 \\
\hline $\mathrm{Pb}$ & $<2$ & 37 & 53 & 31 & 35 & 30 & 5 & 13 & 7 & 5 & 31 & 37 \\
\hline $\mathrm{Ni}$ & 8 & 16 & 17 & 12 & 14 & 13 & 5 & 13 & 40 & 32 & 14 & 12 \\
\hline $\mathrm{Co}$ & 17 & 26 & 26 & 19 & 19 & 14 & 8 & 15 & 17 & 16 & 13 & 20 \\
\hline $\mathrm{Rb}$ & 47 & 311 & 322 & 92 & 231 & 118 & 38 & 168 & 18 & 19 & 56 & 94 \\
\hline $\mathrm{Cr}$ & $<5$ & $<15$ & $<15$ & $<5$ & $<5$ & $<5$ & $<5$ & $<5$ & 70 & 62 & $<5$ & $<5$ \\
\hline $\mathrm{Sr}$ & 34 & 27 & 36 & 14 & 87 & 56 & 14 & 73 & 8 & 14 & 77 & 16 \\
\hline $\mathrm{Zr}$ & 395 & 353 & 444 & 378 & 660 & 410 & 290 & 457 & 197 & 193 & 706 & 387 \\
\hline $\mathrm{Nb}$ & 15 & 27 & 38 & 47 & 13 & 8 & $<5$ & 10 & 8 & 6 & 18 & 51 \\
\hline $\mathrm{Cu}$ & 18 & 13 & 10 & 23 & 1129 & 846 & 7 & 21 & 9 & 14 & 20 & 25 \\
\hline $\mathrm{Zn}$ & 13 & 20 & 42 & 30 & 59 & 147 & 12 & 11 & 20 & 23 & 19 & 30 \\
\hline $\mathrm{Y}$ & 47.3315 & 83.7505 & 129.8468 & 64.9064 & 35.4044 & 35.0653 & 13.8892 & 64.7955 & 26.496 & 29.6307 & 22.5875 & 80.5626 \\
\hline $\mathrm{U}$ & 13.5019 & 23.8973 & 13.9364 & 78.6425 & 12.4444 & 12.7403 & 4.5164 & 14.9985 & 3.0552 & 3.0754 & 2.1289 & 88.1412 \\
\hline $\mathrm{La}$ & 333.8817 & 104.9958 & 101.6811 & 13.3387 & 26.9023 & 11.904 & 9.0593 & 79.9325 & 43.3793 & 80.5753 & 33.0515 & 14.8409 \\
\hline $\mathrm{Ce}$ & 473.6367 & 199.3189 & 206.1465 & 16.2868 & 60.0364 & 29.0596 & 26.2992 & 153.6767 & 110.1687 & 159.8011 & 93.3079 & 17.3756 \\
\hline $\operatorname{Pr}$ & 48.707 & 21.3463 & 23.0739 & 2.7204 & 4.7093 & 2.204 & 1.9055 & 14.2569 & 9.4158 & 17.1025 & 7.9974 & 3.7371 \\
\hline $\mathrm{Nd}$ & 166.8479 & 79.2532 & 89.2223 & 11.9451 & 17.202 & 8.5812 & 6.852 & 52.1876 & 34.5522 & 64.0502 & 29.4054 & 16.4185 \\
\hline $\mathrm{Sm}$ & 25.2098 & 14.7661 & 18.5844 & 4.296 & 3.6908 & 2.2036 & 1.3487 & 10.5229 & 5.8968 & 10.2616 & 4.8465 & 5.8315 \\
\hline $\mathrm{Eu}$ & 4.0533 & 1.5973 & 1.6408 & 0.3865 & 0.9967 & 0.7759 & 0.165 & 2.1375 & 1.2861 & 2.1799 & 1.0912 & 0.5541 \\
\hline $\mathrm{Gd}$ & 20.6259 & 14.0402 & 18.0641 & 4.9456 & 3.7047 & 2.4328 & 1.4351 & 9.8418 & 5.0251 & 8.0105 & 4.2661 & 6.6073 \\
\hline $\mathrm{Tb}$ & 2.6457 & 2.6076 & 3.6578 & 1.4937 & 0.8093 & 0.618 & 0.3098 & 1.9034 & 0.8338 & 1.1667 & 0.7001 & 2.1645 \\
\hline Dy & 9.8925 & 14.7567 & 21.7941 & 11.3967 & 5.1267 & 4.4818 & 2.1491 & 11.6517 & 4.6862 & 5.5034 & 3.8848 & 14.7296 \\
\hline Ho & 1.8306 & 3.1761 & 5.015 & 2.8856 & 1.2444 & 1.2275 & 0.5184 & 2.6671 & 1.0024 & 1.1548 & 0.8597 & 3.6338 \\
\hline Er & 5.1167 & 9.2247 & 14.4437 & 9.1647 & 3.6718 & 3.9102 & 1.4793 & 7.4372 & 2.8293 & 3.2394 & 2.4406 & 10.2959 \\
\hline $\mathrm{Tm}$ & 0.7312 & 1.4615 & 2.4292 & 1.84 & 0.6495 & 0.7454 & 0.2478 & 1.2708 & 0.4323 & 0.4815 & 0.3839 & 2.3585 \\
\hline $\mathrm{Yb}$ & 4.5178 & 9.1866 & 15.1304 & 13.1375 & 4.1781 & 4.9339 & 1.5258 & 7.8969 & 2.722 & 2.9932 & 2.3231 & 17.6225 \\
\hline $\mathrm{Lu}$ & 0.6294 & 1.2832 & 2.1148 & 1.9331 & 0.5725 & 0.7076 & 0.2284 & 1.0741 & 0.38 & 0.3909 & 0.3197 & 2.5427 \\
\hline
\end{tabular}


Table 3. (continued)

\begin{tabular}{|c|c|c|c|c|c|c|c|c|c|c|c|c|}
\hline \multicolumn{13}{|c|}{ Normative mineralsy } \\
\hline & BRS-04 & BRS-46 & BRS-47 & BRS-86 & BRS-90 & BRS-91 & BRS-92 & BRS-100 & BRS-101 & BRS-102 & BRS-103 & BRS-84 \\
\hline $\mathrm{Q}$ & 41.728 & 30.608 & 24.436 & 20.669 & 9.833 & 0 & 25.260 & 31.403 & 0 & 4.789 & 0 & 19.602 \\
\hline $\mathrm{C}$ & 0.866 & 0.000 & 0.558 & 0 & 0 & 0 & 0 & 1.466 & 0 & 0 & 0.872 & 0 \\
\hline Or & 6.087 & 34.572 & 31.499 & 13.533 & 22.870 & 15.483 & 8.214 & 23.166 & 1.477 & 1.596 & 6.678 & 13.829 \\
\hline $\mathrm{Ab}$ & 45.101 & 23.947 & 30.462 & 60.341 & 61.317 & 69.478 & 60.877 & 37.147 & 66.582 & 69.890 & 62.403 & 61.091 \\
\hline An & 1.859 & 1.888 & 4.558 & 0 & 0 & 0 & 0 & 1.198 & 0 & 0 & 2.561 & 0 \\
\hline $\mathrm{Ne}$ & 0 & 0 & 0 & 0 & 0 & 5.563 & 0 & 0 & 3.496 & 0 & 17.901 & 0 \\
\hline Ac & 0 & 0 & 0 & 0.961 & 0.102 & 0.266 & 1.360 & 0 & 4.984 & 4.625 & 0 & 1.642 \\
\hline Ns & 0 & 0 & 0 & 0 & 0 & 0 & 2.094 & 0 & 0 & 0 & 0 & 0 \\
\hline $\mathrm{Di}$ & 0 & 0.428 & 0 & 1.021 & 0.800 & 0.135 & 0 & 0 & 16.664 & 12.788 & 0 & 1.075 \\
\hline Wo & 0 & 0 & 0 & 0.150 & 0 & 0 & 0 & 0 & 0 & 1.376 & 0 & 0.142 \\
\hline Hy & 0.274 & 0.798 & 1.096 & 0 & 0.028 & 0 & 0.075 & 0.822 & 0 & 0 & 0 & 0 \\
\hline $\mathrm{Ol}$ & 0 & 0 & 0 & 0 & 0 & 0.602 & 0 & 0 & 0.434 & 0 & 0.541 & 0 \\
\hline Il & 0.064 & 0.064 & 0.107 & 0.043 & 0.064 & 0.086 & 0 & 0.043 & 0.064 & 0.064 & 0.214 & 0.043 \\
\hline $\mathrm{Hm}$ & 0.860 & 3.990 & 4.480 & 0.908 & 2.085 & 4.468 & 0 & 1.880 & 0.897 & 0.481 & 2.620 & 0.732 \\
\hline Tn & 0 & 0.850 & 0 & 0.141 & 0.727 & 0 & 0.491 & 0 & 0 & 1.537 & 0 & 0.141 \\
\hline $\mathrm{Pf}$ & 0 & 0 & 0 & 0 & 0 & 0.741 & 0 & 0 & 1.083 & 0 & 0 & 0 \\
\hline $\mathrm{Ru}$ & 0.236 & 0 & 0.404 & 0 & 0 & 0 & 0.050 & 0.408 & 0 & 0 & 0.378 & 0 \\
\hline$A p$ & 0.189 & 0.189 & 0.308 & 0.095 & 0.261 & 0.071 & 0.071 & 0.213 & 0.284 & 0.261 & 0.403 & 0.095 \\
\hline Sum & 97.265 & 97.335 & 97.908 & 97.862 & 98.086 & 96.892 & 98.492 & 97.745 & 95.967 & 94.406 & 94.570 & 98.392 \\
\hline
\end{tabular}

TAS (Cox et al. 1979)

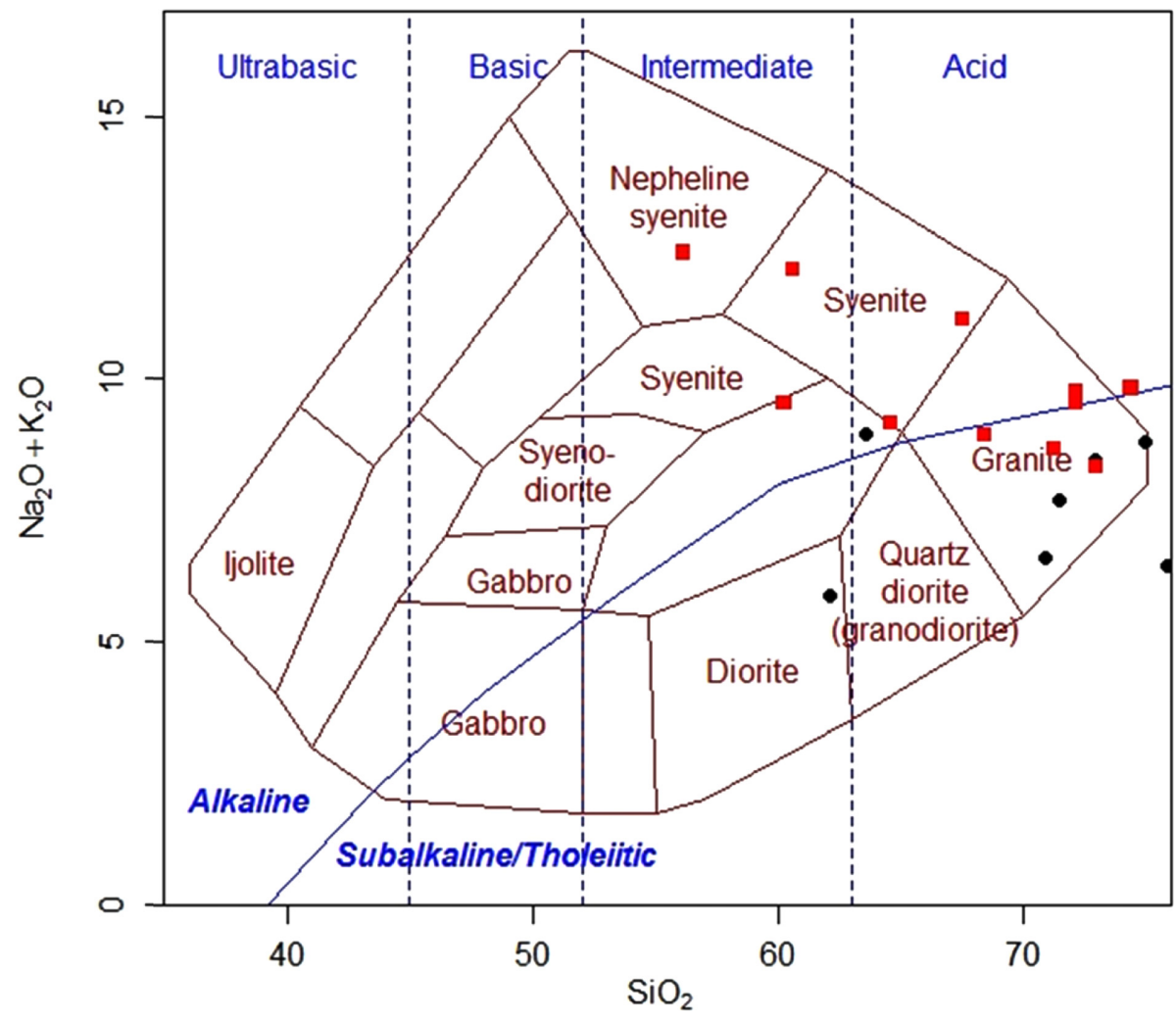

Figure 10. Total alkali vs. silica (TAS) diagram of selected samples of albitite. Red squares are samples from Udaipurwati granite gneiss and black dots are samples from Saladipura-Guhala area.

\section{Geochemistry}

Whole rock chemistry data reveals (Tables 2 and 3) that the albitite samples have variable silica and $\mathrm{CaO}$ content, low $\mathrm{MgO}, \mathrm{TiO}_{2}$, very low $\mathrm{MnO}$ and $\mathrm{P}_{2} \mathrm{O}_{5}$. Average $\mathrm{K}_{2} \mathrm{O} / \mathrm{Na}_{2} \mathrm{O}$ ratio is 0.01 indicating that soda content is higher than potash. In CIPW norm, normative acmite appears in maximum numbers of samples which also indicate hypersodic nature of the albitite. The chemical data also reveals that the albitite veins intruded within Udaipurwati granite gneiss is relatively silica poor than the albitites of Saladipura-Guhala area. In some samples from the veins of Udaipurwati granite gneiss, normative nepheline and olivine appears, indicating silica undersaturated nature. High $\mathrm{K}_{2} \mathrm{O}$ content in some samples of the Udaipurwati granite gneiss, compared to the general low-K nature of the majority of the samples of both the areas is possibly due to contamination by host granite country rock during emplacement of albitite within Udaipurwati granite gneiss. Molar proportion of $\mathrm{Al}_{2} \mathrm{O}_{3}$ to $\mathrm{K}_{2} \mathrm{O}+\mathrm{Na}_{2} \mathrm{O}$ indicates the albitites are metaluminous to peralkaline. In $\mathrm{SiO}_{2}$ vs. $\mathrm{K}_{2} \mathrm{O}+\mathrm{Na}_{2} \mathrm{O}$ diagram (Fig. 10; after Cox et al., 1979) the majority of the samples from the Udaipurwati granite gneiss falls in alkaline 


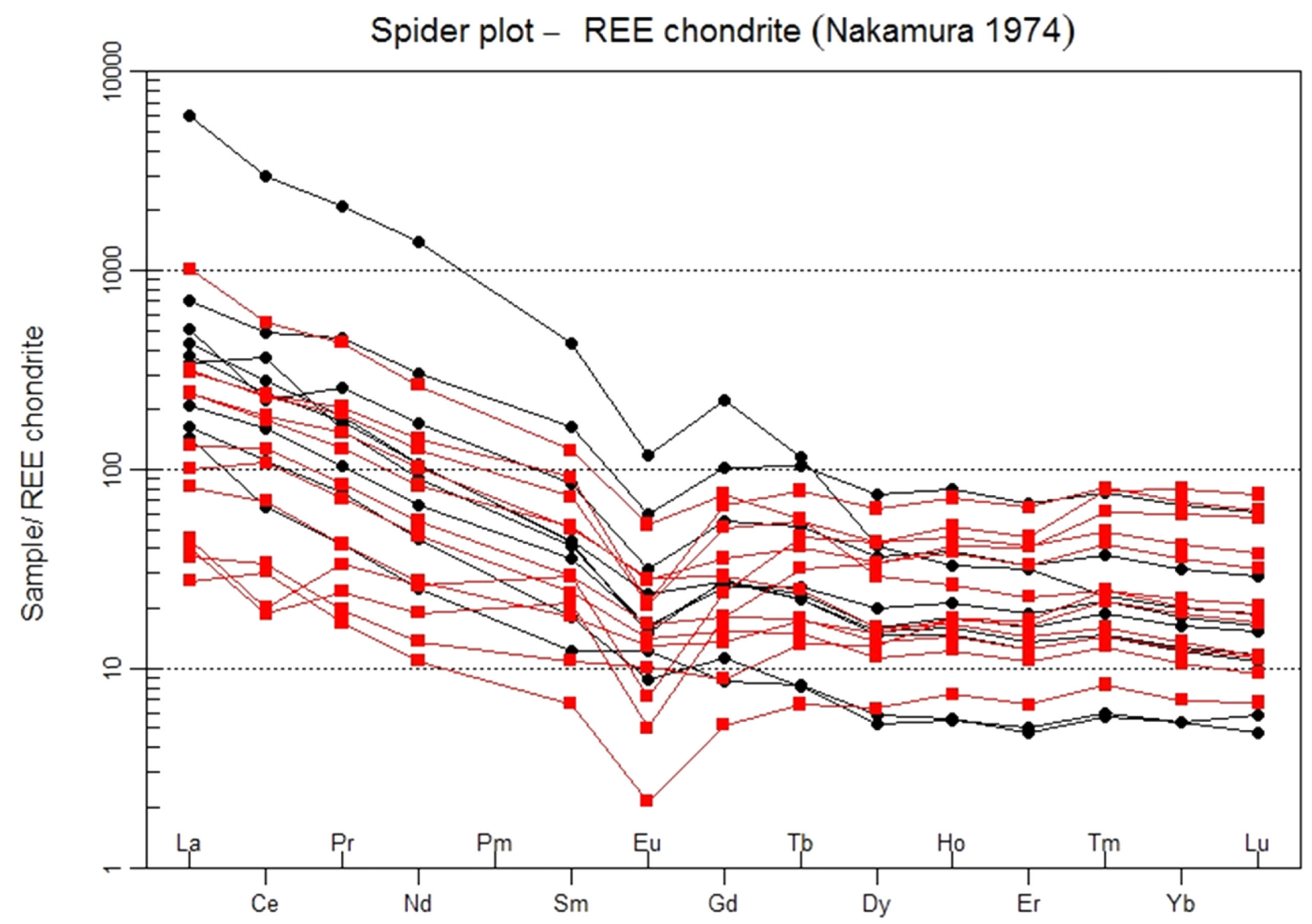

Figure 11. Chondrite normalised REE pattern of albitite (after Nakamura, 1974).

field and classified as syenite to granite whereas the samples from the Saladipura-Guhala area falls in the subalkaline field and classified as granite to diorite.

The REE data (Tables 2 and 3) of the albitite and their chondrite normalized pattern (Fig. 11) show variable degree of REE fractionation, indicated by the ratio $\mathrm{La}_{\mathrm{N}} / \mathrm{Yb}_{\mathrm{N}}=0.56$ to 298.30 . Both LREE and HREE fractionation are more and variable, indicated by the ratios of $\mathrm{La}_{\mathrm{N}} / \mathrm{Sm}_{\mathrm{N}}=1.57$ to 13.98 and $\mathrm{Ce}_{\mathrm{N}} / \mathrm{Sm}_{\mathrm{N}}=0.70$ to 8.78 . Out of twenty one samples, nineteen samples show negative $\mathrm{Eu}\left(\mathrm{Eu} / \mathrm{Eu}^{*}\right.$ value ranging from 0.26 to 0.83 ) anomaly, indicating plagioclase fractionation at source which is further supported by less amount of $\mathrm{CaO}$ and crystallisation in reducing environment. The geochemical data of albitites of both the areas (Saladipura-Guhala and Udaipurwati granite gneiss) indicate that they are cogenetic in nature.

\section{Discussion and Conclusions}

Compositional variability of ecandrewsite from ferroan manganoan ecandrewsite to zincian manganoan ilmenite and their mineral association suggests that they were formed due to replacement of primary magmatic minerals of ilmenite group. The compositional variability within individual mineral grains probably reflects the changes in the alkalinity of the fluid involved. The variations of the soda content shown by appearance of normative acmite, but absence of any sodic amphibole or sodic pyroxene within the albitite indicate involvement of soda rich fluid from outside the system. Core to rim enrichment of $\mathrm{Zn}$ content within ecandrewsite grains also indicate replacement of zinc in later stage. Tables 2 and 3 indicate enrichment of $\mathrm{Cu}, \mathrm{U}$ and REE in the samples of albitite, and the KFB is an established copper producing area in Rajasthan (Ray, 2004). Therefore, it may be possible that along with soda and $\mathrm{Zn}$, many other metals $(\mathrm{Cu}, \mathrm{Au}, \mathrm{U}, \mathrm{REE})$ migrated through mantle derived fluids from outside the system along the deep seated fracture zones ('intracontinental rift' of Ray, 1990) envisaged for the emplacement of the albitites. Zn specially migrated along the grain boundaries of ilmenite through an alkaline fluid along micro-shear zones in the rock and precipitated the element along the grain boundary towards core part by replacing the already existing ilmenite grains in the albitite. This is reflected in core to rim enrichment of Zn content (Table 1, Figs. 5 and 6) within ecandrewsite grains. Occurrence of ecandrewsite, thus, suggest a petrotectonic significance wherein the migration of elements ( $\mathrm{Zn}, \mathrm{Cu}-\mathrm{Au}-\mathrm{U}, \mathrm{REE})$ took place through the major deep seated fractures and associated micro-shears generated during the Proterozoic Khetri fold belt development and emplacement of the Udaipurwati granite pluton. Introduction of $\mathrm{Zn}$ from outside ilmenite also indicate possibility of accumulation of other compatible metals associated with $\mathrm{Zn}$, like $\mathrm{Cu}, \mathrm{Pb}, \mathrm{Au}$ and also $\mathrm{U}$ and REE. This unusual mineral found in the albitite within Udaipurwati granite gneiss may also be an indicator mineral for search of $\mathrm{Cu}-\mathrm{Au}-\mathrm{U}$ and REE metals within the granite gneiss country and possibly diamond within the ultramafic-mafic rock dominated basement part of the KFB. This unusual mineral association of ecandrewsite with ilmenite 
may also be looked into other areas of similar geological set up within NW Indian craton and elsewhere in the world for search of base metals, gold, Uranium and REE of importance.

\section{Acknowledgements}

The authors wish to thank the anonymous reviewers for fruitful suggestions in improving this paper. Sri A. Thiruvengadam, HoD, GSI WR Jaipur is thanked for giving permission to publish this paper.

\section{References}

Brown, R.N., Riley, J.F., and Schultz, R.K., 1970, Contributions to Australasian mineralogy: I. A new zinc-bearing ilmenite from Broken Hill: Amdel Bulletin, no. 10, pp. 48-50.

Birch, W.D., Burke, E.A.J., Wall, V.J., and Etheridge, M.A., 1988, Ecandrewsite, the zinc analog of ilmenite, from Little Broken Hill, New South Wales, Australia, and the San Valentin mine, Sierra de Cartegena, Spain: Mineralogical Magazine, v. 52, no. 365, pp. 237-240.

Cox, K.G., Bell, J.D., and Parkhurst, R.J., 1979, The Interpretation of Igneous Rocks: Chapman \& Hall, London, 450 p.

Dasgupta, S.P., 1968, The structural history of the Khetri Copper Belt, Jhunjhunu and Sikar districts, Rajasthan: Memoir of Geological Survey of India, v. 98, 170 p.

Fareeduddin and Bose, U., 1992, A new occurrence of albitite dyke near Arath NAgaur district, Rajasthan: Current Science, v. 62, no. 9, pp. 635-636.

Guha, D.B., and Mohanty, M., 1994, Occurrence of albitite rocks within the Mangalwar Complex of southcentral Rajasthan,India: Bulletin of the Indian Geologists' Association, v. 27, no. 1, pp. 35-43.

Gupta, P., Chattopadhyay, B., and Guha, D.B., 1998, Basement-cover relationship in the Khetri Copper Belt and the emplacement mechanism of the granite massifs Rajasthan, India: Journal of the Geological Society of India, v. 52, pp. 417-432.

Mitchell, R.H., and Liferovich, R.P., 2004, Ecandrewsite-zincian pyrophanite from Lujavrite, Pilanberg alkaline Complex, South Africa: The Canadian Mineralogist, v. 42, pp. 1169-1178.

Nakamura, N., 1974, Determination of REE, Ba, Fe, Mg, Na and K in carbonaceous and ordinary chondrites: Geochimica et Cosmochimica Acta. v. 38, no. 5, pp. 757-775.

Ray, S.K., 1987, Albitite occurrences and associated ore minerals in the Khetri copper belt North-Eastern Rajasthan: Records of the Geological Survey of India, v. 113, no. 7, pp. 41-49.

Ray, S.K., 1989, The albitite dykes of Dudu sector in Jaipur district, Rajasthan and their tectonic significance: Indian Journal of Geology, v. 61, no. 2, pp. 129-132.

Ray, S.K., 1990, The albitite line of northern Rajasthan - a fossil intracontinental rift zone: Journal of the Geological Society of India, v. 36, pp. 413-423.

Ray, S.K., 2004, Mineral potential of the albitite line of Rajasthan: Geological Survey of India, Special Publication, v. 72, pp. 487-496.

Sakoma, E.M., and Martin, R.F., 2002, Oxidation-induced postmagmatic modifications of primary ilmenite, NYG-related aplite dyke, Tibchi complex, Kalato, Nigeria: Mineralogical Magazine, v. 66, no. 4, pp. 591-604.

Yadav, G.S., Muthamilselvan, A., Shaji, T.S., Nanda, L.K., and Rai, A.K., 2015, Recognition of a new albitite zone in northern Rajasthan: its implications on uranium mineralization: Current Science, v. 10, no. 11, pp. 1994-1998.

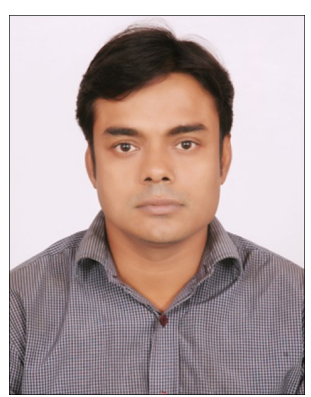

Sandip Neogi is a Senior Geologist of Geological Survey Of India, Govt Of India, Ministry of Mines and presently posted in technical coordination division of State Unit - West Bengal and Andaman Nicobar at Kolkata, West Bengal (India). He did his $\mathrm{MSc}$ in Applied Geology form Presidency College, Kolkata and after pursuing MSc degree he worked as a Junior Research Fellow (1.5 year) in a Department of Science and Technology, Govt Of India sponsored research project related to the evolution of Tista Dome in Sikkim - Darjeelig Himalaya in the same Institute. He has published several paper in various scientific journals and his area of interest and research are structural geology, petrology and geochemistry.

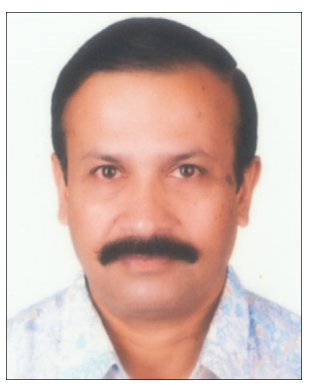

D.B. Guha is presently located at Jaipur, Rajasthan (India) as Deputy Director General (Retired) of Geological Survey of India. Dr. Guha obtained his Graduation (Honours) and Post-Graduation in Geology from Banaras Hindu University, Varanasi, India and did Doctorate research in the Lesser Himalayan metamorphics of Kashmir (India) from Panjab University, Chandigarh (India). He extensively studied the high grade granulites and greenstone cratonic terrane of Rajasthan India. He established sizeable gold deposit in Rajasthan, India. He has to his credit more than forty original research papers. He has been awarded with National Mineral Award-2005 by Government of India, B.P.Radhakrishna Prize of Geological Society of India, and Geological Mining \& Metallurgical Society's silver medal for his geological findings.

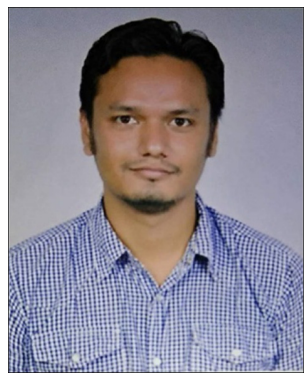

Prateek Garkhal is a Geologist of Geological Survey of India, Govt. of India and presently posted in Mineral Exploration division of State Unit-Rajasthan at Jaipur, Rajasthan (India). He did his M.Sc. in Geology from University of Rajasthan, Jaipur, India. He also worked on $\mathrm{PhD}$ "Geology and Geochemistry of Mafic and Ultramafic rocks from SE of Bidasar, Churu district, Rajasthan" as a Research Scholar under supervision of Prof. M.K. Pandit in Department of Geology, University of Rajasthan, Jaipur, India. He has experience in Specialised Thematic Geological Mapping, Geochemical Mapping and Mineral Exploration. 